Meta

Journal des traducteurs

Translators' Journal

\title{
Direccionalidad y formación de traductores: un estudio longitudinal de los procesos cognitivos en la traducción inversa
}

\section{Nadia Rodríguez et Bettina Schnell}

Volume 57, numéro 1, mars 2012

La CIUTI, chef de file pour la promotion de l'employabilité et de la recherche

CIUTI: Leader in Advocating Employability and Research

URI : https://id.erudit.org/iderudit/1012741ar

DOI : https://doi.org/10.7202/1012741ar

Aller au sommaire du numéro

Éditeur(s)

Les Presses de l’Université de Montréal

ISSN

0026-0452 (imprimé)

1492-1421 (numérique)

Découvrir la revue

Citer cet article

Rodríguez, N. \& Schnell, B. (2012). Direccionalidad y formación de traductores: un estudio longitudinal de los procesos cognitivos en la traducción inversa.

Meta, 57(1), 67-81. https://doi.org/10.7202/1012741ar
Résumé de l'article

Le présent article expose les fondements théoriques et la conception d'une étude empirique longitudinale relatives au processus de traduction. L'objectif de la recherche est de mieux comprendre les processus traductionnels utilisés par les étudiants de premier cycle lorsqu'ils traduisent de leur langue maternelle L1 (l'espagnol) vers leur seconde langue L2 (le français ou l'allemand). Afin de mieux comprendre comment les compétences de traduction en L2 se développent et comment elles peuvent être dynamisées, le comportement traductionnel des étudiants a été analysé à des moments précis de leur formation, c'est-à-dire en deuxième année et en troisième année, juste avant de conclure leurs études. L'étude vise à déterminer comment les sujets abordent l'acte de traduction (macrostratégie), quelles stratégies ils emploient pour trouver des solutions provisoires aux problèmes de traduction rencontrés, dans quelle mesure ils sont capables d'activer des routines de traduction ainsi que des procédés standards de transfert spécifiques de la combinaison linguistique en jeu et, enfin, comment ces habiletés se développent au cours du temps. Les résultats obtenus devraient clarifier le mode de déploiement des compétences en traduction et donc faciliter la mise en oeuvre de formations fondées sur les processus. Une telle approche se révèle nécessaire en raison du défi représenté par l'Espace européen de l'éducation supérieure, qui a entraîné le passage d'une formation centrée sur le professeur à une formation centrée sur l'apprenant, qui priorise le développement des compétences des étudiants. Cette méthodologie fondée sur les processus invite les étudiants à réfléchir sur leur comportement traductionnel et favorise le développement des stratégies métacognitives qui, en fin de compte, contribuent à l'acquisition d'une compétence professionnelle affirmée.
Ce document est protégé par la loi sur le droit d'auteur. L'utilisation des services d’Érudit (y compris la reproduction) est assujettie à sa politique d'utilisation que vous pouvez consulter en ligne.

https://apropos.erudit.org/fr/usagers/politique-dutilisation/ 


\title{
Direccionalidad y formación de traductores: un estudio longitudinal de los procesos cognitivos en la traducción inversa
}

\author{
NADIA RODRÍGUEZ \\ Universidad Pontificia Comillas, Madrid, España \\ nrodiguez@chs.upcomillas.es
}

BETTINA SCHNELL

Universidad Pontificia Comillas, Madrid, España

bschnell@chs.upcomillas.es

\begin{abstract}
RÉSUMÉ
Le présent article expose les fondements théoriques et la conception d'une étude empirique longitudinale relatives au processus de traduction. L'objectif de la recherche est de mieux comprendre les processus traductionnels utilisés par les étudiants de premier cycle lorsqu'ils traduisent de leur langue maternelle L1 (l'espagnol) vers leur seconde langue L2 (le français ou l'allemand). Afin de mieux comprendre comment les compétences de traduction en $\mathrm{L} 2$ se développent et comment elles peuvent être dynamisées, le comportement traductionnel des étudiants a été analysé à des moments précis de leur formation, c'est-à-dire en deuxième année et en troisième année, juste avant de conclure leurs études. L'étude vise à déterminer comment les sujets abordent l'acte de traduction (macrostratégie), quelles stratégies ils emploient pour trouver des solutions provisoires aux problèmes de traduction rencontrés, dans quelle mesure ils sont capables d'activer des routines de traduction ainsi que des procédés standards de transfert spécifiques de la combinaison linguistique en jeu et, enfin, comment ces habiletés se développent au cours du temps. Les résultats obtenus devraient clarifier le mode de déploiement des compétences en traduction et donc faciliter la mise en œuvre de formations fondées sur les processus. Une telle approche se révèle nécessaire en raison du défi représenté par l'Espace européen de l'éducation supérieure, qui a entraîné le passage d'une formation centrée sur le professeur à une formation centrée sur l'apprenant, qui priorise le développement des compétences des étudiants. Cette méthodologie fondée sur les processus invite les étudiants à réfléchir sur leur comportement traductionnel et favorise le développement des stratégies métacognitives qui, en fin de compte, contribuent à l'acquisition d'une compétence professionnelle affirmée.
\end{abstract}

\section{ABSTRACT}

The article outlines the theoretical foundations and the design of a longitudinal study within the field of translation process research. The aim of this empirical study is to get a better understanding of the translation processes deployed by undergraduate students when translating from their mother tongue (Spanish) into their second language (French or German). In order to gain more in-depth insight into how L2 translation competence develops and can best be fostered, students' translation behavior is being monitored at different points of time during their course of studies: in the second year and third year of their undergraduate program, shortly before graduation. The study aims to identify how the subjects approach the translation task (macrostrategy), what kind of strategies they use in order to create tentative solutions to translation problems, to what extent they are able to activate translation routines and language-pair specific standard transfer operations and how these abilities develop over time. The results of this study will hopefully 
provide insight into the unfolding of translation skills and thus allow for the development of a process-based approach to translator training. This will become a necessity against the background of the challenges of the European Space of Higher Education, which has produced a shift in university education from a teacher-centered to a learner-centered approach, where special emphasis is placed on the development of student's skills. Such a process-based methodology invites students to reflect on their translation behavior and fosters the development of metacognitive strategies which ultimately contribute to a competent and successful professional performance.

\section{MOTS-CLÉS/KEYWORDS}

processus de traduction, étude longitudinale, compétence traductionnelle, stratégie traductionnelle, formation

translation process, longitudinal study, translation competence, translation strategies, training

\section{Investigación de los procesos de traducción: el estado de la cuestión}

En el ámbito de la traductología la aplicación del paradigma cognitivo se remonta a los años 1980 cuando, impulsado por los estudios en el campo del aprendizaje de segundas lenguas, la investigación deja de centrarse exclusivamente en el análisis del producto, es decir, la traducción, para orientarse hacia los procesos mentales subyacentes a partir de la observación y el análisis del comportamiento del traductor. Con los primeros estudios de carácter exploratorio, entre los que el trabajo de Krings (1986) es referencia obligada, la investigación traductológica se ha tornado empírica y experimental.

Desde su inicio, la investigación de los procesos de traducción ha sido un campo muy dinámico que ha producido un gran número de estudios y publicaciones. Una discusión detallada de las investigaciones realizadas trasciende el alcance y la intención del presente artículo. Sin embargo es preciso apuntar que en los últimos años la investigación del proceso de traducción gira en torno a una serie de cuestiones emergentes. Entre éstas se encuentran, la incidencia de factores externos sobre el proceso de traducción, como la dimensión afectiva, la presión de tiempo (Bayer-Hohenwarter 2008) y el impacto de la utilización de memorias de traducción (O’Brien 2006; 2008; 2011; Aranda Novillo 2011), el análisis de fases específicas del proceso de traducción, como las fases de comprensión y de producción (Dragsted y Gorm Hansen, 2008) o la de la revisión (Künzli 2006; 2007), la pericia y las diferencias entre el comportamiento traductor entre estudiantes de traducción y profesionales (Englund Dimitrova 2005; Künzli 2001; 2004; 2005), así como el desarrollo de la competencia traductora (PACTE 2002; 2005; 2007). La investigación de los procesos de traducción no solamente se ha ido orientando hacia problemas cada vez más específicos, sino que, también el nivel de sofisticación de los diseños experimentales, así como el de las técnicas de recogida de datos ha aumentado considerablemente. La metodología tradicional basada en la obtención de datos verbales de los sujetos mediante la introspección a través de think-aloud protocols (TAP) individuales, dialogados o grupales o mediante la retrospección ha sido complementada con un amplio elenco de instrumentos de recogida de datos entre los que cuentan entrevistas y encuestas, el protocolo de traducción en el que se registran las acciones del teclado y que permite controlar y visualizar a través de indicadores procesuales y estadísticos variaciones de tiempo, pausas, correcciones, etc., observadas a lo largo del proceso de traducción, 
la grabación en vídeo, así como el seguimiento ocular para determinar exactamente en qué partes de la pantalla y del texto origen el sujeto fija la vista, y, por tanto su atención. Más recientemente se han incorporado las nuevas técnicas en imagología como la tomografía de resonancia magnética funcional (fMRT), o la tomografía por emisión de positrones (PET) para visualizar los procesos cerebrales que cobran especial relevancia en la investigación del proceso de interpretación.

Como cada uno de estos métodos tiene sus ventajas y sus limitaciones, las investigaciones más recientes tienden a recurrir a una metodología múltiple, basada en la combinación de instrumentos de recogida de datos complementarios que permiten la triangulación de los resultados. Aparte de la metodología, otro problema intrínseco de la investigación de los procesos de traducción es la validez externa de los resultados debido al tamaño reducido de las muestras. El tamaño de las muestras es un problema difícil de solucionar debido a la complejidad de los diseños experimentales, la profundidad de análisis de los datos, así como el tipo de tarea encomendada que requiere una participación prolongada de los sujetos. La falta de recursos económicos y humanos dificulta la realización de estudios a gran escala, de modo que la réplica de estudios en distintas instituciones se perfila como un enfoque empírico que permite lograr resultados extrapolables.

\section{Direccionalidad: Investigación de la traducción inversa}

En la traductología occidental ha prevalecido durante largo tiempo el concepto ideológico de la supremacía de la traducción directa, quedando deslegitimado el ejercicio profesional de la traducción inversa. Por consiguiente, el estudio de la traducción inversa ha sido un aspecto ampliamente desatendido por parte de la investigación traductológica hasta hace relativamente poco.

Han sido autores como Kiraly (1995), Campbell (1998) y Prunč (2000) que han propuesto una reflexión crítica sobre la supremacía de la traducción directa desde una perspectiva teórica, partiendo de una concepción de la competencia traductora, no basada de forma apriorística en la noción del dominio absoluto de la lengua materna. Su crítica gira principalmente en torno al dominio de la lengua materna como eje central de la competencia traductora, ya que este modo de plantear la competencia traslativa encierra una doble falacia: presupone que el traductor que traduce a su L1 tiene una competencia absoluta de su propia lengua y, además, subestima la importancia de la comprensión del TO para la traducción a la lengua materna. Pero también, apunta Prunč (2000), la noción de la competencia lingüística absoluta del hablante nativo necesita ser revisada, teniendo en cuenta que la competencia en la lengua materna no es necesariamente la competencia más desarrollada y que la competencia lingüística en L1 suele variar considerablemente de un ámbito lingüístico especializado a otro.

Sin embargo, en los últimos años la cuestión de la direccionalidad ha ido cobrando cada vez mayor relevancia en la investigación traductológica debido a la globalización del mercado de traducción y la predominancia del inglés en el ámbito científico y profesional que convierte la traducción hacía una lengua no materna en una práctica frecuente y no sólo en los países de lenguas minoritarias. En este sentido se expresa Pavlović cuando afirma: 
In spite of the 'Golden Rule' that translators should only work into their first language, translation into the second language (L2 translation) is a fact of life in settings involving languages of 'limited diffusion'. Even in countries that use one of the traditionally 'major' languages, research into L2 is becoming increasingly topical with the emergence of global translation markets and the worldwide dominance of English. (Pavlović 2010a: 63)

Sin duda alguna, la investigación de la direccionalidad en traducción ha recibido fuertes impulsos por parte de investigadores de países con lenguas de menor difusión (Kocijančič Pokorn 2000; 2005; Pavlović 2010a; 2010b), pero no se ciñe exclusivamente a ellos, tal y como lo demuestran las publicaciones de Kelly, Nobs et al. (2003) y Kelly (2005) y el grupo de investigación PACTE de la Universidad Autónoma de Barcelona.

\section{Direccionalidad, investigación del proceso de traducción y enseñanza de la traducción}

En la última década se ha manifestado no solamente un creciente interés académico por la direccionalidad, sino que, además, se ha producido una convergencia entre la investigación del proceso de traducción, la direccionalidad y la enseñanza de la traducción, impulsada también por la implementación del Espacio Europeo de Educación Superior que ha provocado/conllevado el cambio de un modelo de enseñanza centrado en el profesor hacia un modelo de aprendizaje centrado en el alumno y orientado hacia el desarrollo de competencias genéricas y específicas. Partiendo, pues, de la premisa que la traducción hacia la L2 es una práctica habitual, se plantea la cuestión de cómo capacitar a los alumnos para afrontar con éxito esta tarea. Para dar una respuesta a esta incógnita es necesario analizar las similitudes y diferencias de la traducción hacia la L1 y la L2 con el fin de averiguar cuáles son las dificultades intrínsecas de la traducción hacia la L2 y cómo éstas se manifiestan. Asimismo, es imprescindible desvelar cómo los alumnos generan soluciones provisionales para los problemas de traducción encontrados, cómo llevan a cabo el proceso de toma de decisiones, etc.

De ahí surge la necesidad de estudios empíricos del proceso de traducción de los alumnos en formación para desarrollar una metodología que presta atención a los aspectos procesuales, ya que este tipo de enfoque pedagógico, tal y como señala González Davies (2005: 74), no solamente mejora la autoconfianza del alumno, sino que, además, contribuye a una mayor coherencia, calidad y productividad en las traducciones. Pavlović comparte esta visión y llega a la siguiente conclusión:

Process-oriented translation pedagogy has the advantage of focusing on procedural, rather than declarative knowledge, and the outcome of such learning lends itself to applicability to new situations. It allows teachers to see what the students are struggling with and thus makes it easier to provide help and advice through relevant projects and tailor-made exercises. (Pavlović 2010a: 65)

\section{Estudio longitudinal: diseño experimental}

Es en este marco en el que confluyen la direccionalidad, el proceso y la didáctica que se inscribe el presente estudio longitudinal. El objetivo principal reside en la obser- 
vación y el análisis del comportamiento traductor de alumnos de traducción a largo plazo para arrojar luz sobre los procesos cognitivos y las estrategias subyacentes, y cómo éstos se modifican y evolucionan a medida que los sujetos avanzan en sus estudios y adquieren mayor competencia y pericia. Los datos observacionales recogidos en distintos momentos de su formación permitirán establecer cómo se desarrolla la competencia traductora de los sujetos. La principal razón que nos ha llevado a emprender este estudio es la relativa carencia de estudios longitudinales con un seguimiento del mismo grupo experimental durante un tiempo prolongado para verificar los modelos existentes de la competencia traductora y de su adquisición (PACTE 2000: 104). Hasta la fecha se ha realizado un único estudio longitudinal en el sentido estricto de la palabra, entendiéndose por ello, siguiendo a Göpferich y Jääskelainen:

By longitudinal studies in the strictest sense of the term we mean investigations into the development of translation competence analyzing the translation behavior (1) of the same subjects (2) at regular intervals (3) over a longer period of time. (Göpferich y Jääskelainen 2009: 183)

Se trata del proyecto Transcomp de la universidad de Graz con un grupo experimental configurado por un total de 12 sujetos. En este sentido, el presente estudio que presentamos en este artículo aspira a ser una modesta contribución a esta línea de investigación.

\subsection{Planteamiento experimental}

El planteamiento experimental se sustenta sobre el modelo de competencia traductora desarrollado por el grupo PACTE (2000) y se organiza en tres fases: una experiencia piloto previa, la recogida de datos del estudio longitudinal en sí y el análisis de los datos obtenidos. El estudio se lleva a cabo con alumnos de la Licenciatura en Traducción e Interpretación de la Universidad Pontificia Comillas que se han brindado voluntariamente. El estudio se realiza en distintas etapas de la formación de los alumnos: al principio de la formación académica (segundo curso académico), cuando los estudiantes se inician en la traducción inversa, cuando hayan adquirido cierta destreza en el ejercicio de la traducción inversa (tercer curso académico) y cuando se hayan convertido en aprendices avanzados (cuarto curso académico).

La primera fase del estudio longitudinal se inició en el año académico 2009/2010 y actualmente (curso académico 2010/2011) hemos concluido la segunda fase de recogida de datos, quedando la tercera fase para el año académico 2011/2012. Una vez concluida la recogida de datos, esperamos poder poner a disposición de la comunidad científica los resultados del estudio a lo largo del curso académico 2012/2013.

\subsection{Selección de los sujetos}

En el presente estudio longitudinal participan un total de 30 alumnos que cursan la Licenciatura en Traducción e Interpretación (cuatro años) en la Universidad Pontificia Comillas. Todos los alumnos iniciaron sus estudios en el curso académico 2008/2009 de modo que en el momento de participar en la primera parte del estudio, estaban concluyendo el segundo cuatrimestre de su segundo año de formación. Este dato 
resulta significativo para el estudio ya que el plan de estudios contempla en segundo curso una estancia de un cuatrimestre en una universidad extranjera, así como una asignatura anual de introducción a la traducción inversa en lengua C. De los 30 sujetos que conforman el grupo experimental, 24 han elegido la combinación lingüística inglés (lengua B) y francés (lengua C) y 6 sujetos restantes la combinación lingüística inglés (lengua $\mathrm{B}$ ) y alemán (lengua $\mathrm{C}$ ).

Es importante señalar que en el momento de participar en la primera parte del estudio 19 de los sujetos habían cursado un cuatrimestre en una universidad de habla francesa o alemana con el fin de mejorar su competencia lingüística en lengua C. Asimismo, todos los sujetos habían recibido durante al menos un cuatrimestre enseñanza en traducción general inversa, de modo que estaban familiarizados con la traducción hacía su lengua C. Si bien la participación en el estudio longitudinal es voluntaria, ésta se realiza en el marco de las asignaturas de traducción inversa al francés y alemán por lo que prácticamente la totalidad de los alumnos que cursan dichas asignaturas accedieron a someterse al experimento.

\subsection{El entorno}

Ambos experimentos realizados hasta el momento tuvieron lugar en una sala de ordenadores de la Universidad Pontificia Comillas. Esto se debe, por una parte, al elevado número de sujetos que participan y que impide contemplar la posibilidad de que los sujetos puedan llevar a cabo la tarea de traducción en su propio domicilio con la herramienta informática elegida. Por otra parte, dada la alta carga lectiva de los alumnos de licenciatura era necesario realizar el experimento en el horario lectivo, solicitando a los profesores que pusieran a disposición un bloque de 2 horas en el horario semanal.

\subsection{Selección de los textos origen}

Para este estudio longitudinal hemos optado por seleccionar distintos textos origen para los experimentos consecutivos con el fin de evitar un efecto de aprendizaje, ya que las tareas de traducción que tienen que ejecutar los sujetos son muy similares. De esta manera, se puede asegurar que los aspectos hallados en el comportamiento traductor no se deben al hecho de haber realizado la misma prueba de traducción con anterioridad.

En la selección de los textos origen se han tenido en cuenta la formación recibida por parte de los sujetos en traducción inversa de lengua C (francés y alemán), así como el tiempo estipulado para la realización de la tarea de traducción. Los textos elegidos debían ser obligatoriamente de carácter general, puesto que los sujetos solamente habían cursado la asignatura Introducción a la traducción general inversa, y aún no están familiarizados con la traducción especializada. En segundo lugar, era determinante elegir un texto origen con una longitud en torno a 350 palabras, siendo éste el número de palabras que los profesores de traducción inversa exigen a los alumnos en los exámenes de traducción inversa, otorgándoles para este tipo de tarea un espacio de tiempo de dos horas.

Con el fin de ajustarnos a los criterios que priman en la formación de la traducción inversa en la Universidad Pontificia Comillas y que son habituales para los 
sujetos, nos basamos en dos textos de carácter divulgativo, publicados en la prensa española que versan sobre temáticas cercanas, concretamente la historia de la arroba y la historia de la patata (véanse anexos 3 y 4). Dado que la tarea de traducción debía realizarse en un tiempo máximo de 2 horas, era necesario modificar la longitud de los textos originales para que no superasen una longitud de 350 palabras, y adaptarlos de manera que presentaran una macroestructura textual análoga.

\subsection{El proceso experimental}

Previo al primer experimento se celebró una primera sesión informativa para informar a los sujetos sobre la finalidad y el desarrollo del experimento, así como el carácter de su participación como sujeto. Dado que el estudio experimental se realizó en el marco de las asignaturas de traducción inversa de la licenciatura y con el fin de aumentar en la medida de lo posible la validez ecológica del experimento se informó a los participantes que su texto meta no sería en ningún caso objeto de evaluación y calificación por parte del profesor.

En una segunda sesión informativa, los sujetos recibieron una breve introducción al funcionamiento del programa de registro de actividad periférica Translog para poder familiarizarse con la herramienta y realizar una pequeña experiencia piloto.

En una segunda fase, se convocaron los sujetos para someterse al experimento, con el encargo de realizar una traducción, manteniendo la función textual del texto origen en el texto meta, es decir, confeccionando una traducción que pueda ser publicada en la prensa para un público de habla francesa o alemana. En cuanto a la ejecución de la tarea, los sujetos disponían de las 2 horas estipuladas, sin que se sumara otro límite temporal relativo a la visualización del TO en el programa Translog para no incrementar la presión ejercida sobre los sujetos y no condicionar el comportamiento traductor global mediante una visualización segmentada del TO.

Una vez concluida la tarea encomendada, se realizó después de ambos experimentos una encuesta que recogía además de los datos personales de los sujetos, información sobre estancias en el extranjero, número de años de enseñanza recibida en lengua $\mathrm{C}$, enseñanzas de teoría de traducción cursadas, la macroestrategia de traducción, las dificultades de traducción encontradas, así como su percepción de su competencia traductora en traducción inversa.

El tercer y último experimento se llevará a cabo en abril 2012 a finales del cuarto curso académico, y se dará por concluido con unas entrevistas de retrospección inducida, en las que se analizarán con cada uno de los sujetos los protocoles de traducción generados en los tres experimentos.

A continuación los investigadores procederán al análisis cualitativo y cuantitativo de los datos obtenidos en el estudio longitudinal.

\subsection{Metodología de recogida de datos}

La metodología de recogida de datos descansa sobre la herramienta de registro de actividad periférica Translog que permite visualizar a través de indicadores procesuales y estadísticos las acciones del teclado, consultas documentales, modificaciones, así como variaciones de tiempo, pausas, etc. observadas a lo largo del proceso de traducción de los sujetos. 
Este instrumento de recogida de datos se complementa con una encuesta realizada a los sujetos después de haber concluido cada uno de los experimentos, así como una retrospección inducida a partir de los tres protocolos de traducción generados por cada sujeto.

\subsection{Hipótesis}

Para el estudio se adopta el supuesto de partida que el comportamiento traductor de los alumnos de traducción a largo plazo arroja luz sobre los procesos cognitivos y estrategias subyacentes, y cómo éstos se modifican y evolucionan a medida que los sujetos avancen en sus estudios y adquieran mayor competencia y pericia. Los datos observacionales recogidos en distintos momentos de su formación permitirán establecer cómo se desarrolla la competencia traductora de modo que se podrá determinar qué factores inciden en la adquisición de habilidades y destrezas de traducción y en qué fases se estructura su desarrollo. Sobre la base de este supuesto de partida, se plantean las siguientes cuestiones, o hipótesis derivadas:

Por último, cuando la lista es parte de una cita, el nombre del autor se indica al final de la siguiente forma:

1) Existirán diferencias en el proceso traductor de los sujetos dependiendo del momento en el que se someten al experimento.

2) El registro de la actividad periférica y los datos obtenidos de las encuestas y de la retrospección inducida permitirán sacar conclusiones sobre la forma de afrontar la tarea traslativa, las estrategias de toma de decisiones y resolución de problemas.

3) Variará la forma de afrontar la tarea de traducción en función de la competencia de traducción adquirida por cada sujeto a lo largo de la formación.

4) La aprehensión del comportamiento traductor durante un tiempo prolongado, combinado con el análisis de sus productos (traducciones) ilustrará sobre el desarrollo de la competencia traductora y contribuirá a trazar una línea de progresión en el aprendizaje de los sujetos.

5) Se podrá diferenciar entre patrones de comportamiento traductor ideosincrásicos y prototípicos del nivel de pericia de los sujetos.

\subsection{Resultados esperados}

Con este estudio esperamos fomentar la toma de conciencia metacognitiva de los alumnos en torno al proceso de traducción que les invita a monitorear su propia actuación lo que puede desembocar en una autorregulación y optimización del proceso traductor.

Además, esperamos poder establecer pautas metodológicas y desarrollar ejercicios y materiales específicos que contribuyan a ejercitar y estimular la competencia traductora en L2.

\section{A modo de conclusión}

El propósito del presente artículo reside ante todo en la presentación del estudio longitudinal que se está llevando a cabo en el Departamento de Traducción e Interpretación de la Universidad Pontificia Comillas. A nuestro entender, la complejidad intrínseca a la traducción hacia una lengua no materna hace necesario realizar 
estudios de esta índole con el fin de poder modelizar con mayor exactitud la adquisición de la competencia traductora en L2 por parte de los alumnos con el fin de establecer una metodología orientada a salvar los escollos específicos que ésta presenta. Es de suma importancia que el alumno traductor tome conciencia de su proceso de traducción para autorregular y optimizar su actuación.

A partir de los resultados del estudio esperamos poder poner de manifiesto la necesidad de incidir en el proceso de aprendizaje de los alumnos con el fin de que desarrollen las estrategias cognitivas adecuadas para garantizar un trabajo de traducción de calidad óptima.

\section{REFERENCIAS BIBLIOGRÁFICAS}

Aranda Novillo, Marcos (2011): La influencia del uso de herramientas de Traducción Asistida por ordenador sobre la calidad y la creatividad. Un estudio experimental del proceso traductológico. Tesis de doctorado no publicada. Madrid: Universidad Pontificia Comillas.

Bayer-Hohenwarter, Gerrit (2008): Alles nur eine Frage der Zeit? Methodologische Überlegungen zu Zeit(Druck) und Translation. Jostrans The Journal of Specialised Translation. 9:108-131. Consultado el 15 de junio de 2011, <http://www.jostrans.org/issue09/art_bayer. php $>$.

CAMPBELl, Stewart (1998): Translation into the Second Language. London/New York: Longman.

Dragsted, Barbara y Gorm Hansen, Inge (2008): Comprehension and production in translation: A Pilot Study on Segmentation and the Coordination of Reading and Writing Processes. In: Susanne Göpferich, Arnt Lykke Ja Kobson y Inger M. Mees, eds. Looking at Eyes. Eye-Tracking Studies of Reading and Translation Processing. Copenhagen Studies in Language 36. Frederiksberg: Samfundslitteratur, 9-29.

Englund Dimitrova, Birgitta (2005): Expertise and Explicitation in the Translation Process. Amsterdam/ Philadelphia: John Benjamins.

González Davies, María (2005): Minding the Process, Improving the Product: Alternatives to Traditional Translator Training. In: Martha Tennant, ed. Training for the New Millennium: Pedagogies for Translation and Interpreting. Amsterdam/Philadelphia: John Benjamins, 67-82.

Göpferich, Susanne y JäÄskeläInen, Riitta (2009): Process Research into the Development of Translation Competence: Where are We, and Where Do We Need to Go? Across Languages and Cultures. 10(2):169-191.

KeLly, Dorothy (2005): The Wrong Way Round? Consideraciones sobre la cuestión de la direccionalidad en la traducción profesional y la formación de traductores. In: Cristina García DE Toro e Isabel García IzQuierdo, eds. Experiencias de traducción. Reflexiones desde la práctica traductora. Castelló de la Plana: Servicio de Publicaciones de la Universitat Jaume I, 129-146.

Kelly, Dorothy, Nobs, Maria L. y SÁnchez, Dolores, eds. (2003): La direccionalidad en Traducción e Interpretacion. Granada: Atrio.

Kiraly, Donald C. (1995): Pathways to Translation. Pedagogy and Process. Kent/Ohio: Kent State University Press.

KociJančič PoKorn, Nike (2000): Translation into a Non-Mother Tongue in Translation Theory: Deconstruction of the Traditional. In: Andrew Chesterman, Natividad Gallardo San Salvador and Yves Gambier eds. Translation in Context: Selected contribution from the EST Congress Granada 1998. Amsterdam/Philadelphia: John Benjamins, 61-72.

KociJančič Pokorn, Nike (2005): Challenging the Traditional Axioms. Translation into a NonMother Tongue. Amsterdam/Philadelphia: John Benjamins.

KRINGS, Hans P. (1986): Was in den Köpfen von Übersetzern vorgeht. Eine empirische Untersuchung zur Strukutur des Übersetzungsprozesses an fortgeschrittenen Französischlernern. Tübingen: Gunter Narr. 
KüNZLI, Alexander (2001): Experts versus novices: l'utilisation de sources d'information pendant le processus de traduction. Meta. 46(3):507-523.

KüNZLI, Alexander (2004): Risk Taking: Trainee Translators vs. Professional Translators. Jostrans The Journal of Specialised Translation. 2:34-49. Consultado el 10 de abril de 2009, <http:// www.jostrans.org/issue02/art_kunzli.php>.

KünZli, Alexander (2005): Investigating Translation Proficiency - Study of the Knowledge Employed by Two Engineers in the Translation of a Technical Text. Bulletin suisse de linguistique appliquée. 81:41-56.

KünZLI, Alexander (2006): Translation Revision: A Study of the Performance of 10 Professional Translators Revising a Technical Text. In: Maurizio Gotti y Susan SARCevic, eds. Insights into Specialized Translation. Frankfurt: Peter Lang, 195-214.

KüNZLI, Alexander (2007): The Ethical Dimension of Translation Revision. An Empirical Study. Jostrans Journal of Specialised Translation. 8:42-56. Consultado el 25 de abril de 2008, $<$ http://www.jostrans.org/issue08/art_kunzli.php $>$.

O'Brien, Sharon (2006): Eye-tracking and Translation Memory Matches. Perspectives: Studies in Translatology. 14(3):185-205.

O'BRIEN, Sharon (2008): Processing fuzzy matches in translation memory tools: an eye-tracking analysis. In: Susanne Göpferich, Arnt Lykke Jaковson e Inger M. Mees, eds. Looking at Eyes: Eye-Tracking Studies of Reading and Translation Processing. Copenhagen Studies in Language. Frederiksberg: Samfundslitteratur, 79-102.

O'Brien, Sharon (2011): Cognitive Explorations of Translation. London: Continuum.

PACTE (2000): Acquiring Translation Competence: Hypotheses and Methodological Problems in a Research Project. In: Allison Beeby, Doris, Ensinger and Marisa Presas, eds. Investigating Translation. Amsterdam: John Benjamins, 99-106.

PACTE (2002): Exploratory Texts in a Study of Translation Competence. Conference Interpretation and Translation. 4(4):41-69.

PACTE (2005): Investigating Translation Competence: Conceptual and Methodological Issues. Meta. 50(2):609-619.

PACTE (2007): Zum Wesen der Übersetzungskompetenz - Grundlagen für die experimentelle Validierung des Ük-Modells. In: Gerd WотנAк, ed. Quo vadis Translatologie? Ein halbes Jahrhundert universitäre Ausbildung von Dolmetschern und Übersetzern in Leipzig. Rückschau, Zwischenbericht und Perspektiven aus der Außenansicht. Berlin: Franck \& Timme, 37-342.

PAVlović, Nataša (2010a): What Were They Thinking?! Student' Decision Making in L1 and L2 Translation Process. Hermes: Journal of Language and Communication Studies. 44:63-87.

Pavlović, Nataša (2010b): Directionality in Translating and Interpreting Practice. Report on a questionnaire in Croatia. 79-95. Consultado el 23 de junio de 2011, <http://isg.urv.es/library/ papers/PavlovicDirectionality.pdf>.

PRUNČ, Erich (2000): Translation in die Nicht-Muttersprache und Translationskultur. In: Meta Grosman, Mirae Kadric, Irena Kovačič, et al., eds. Translation into Non-Mother Tongues in Professional Practice and Training. Tübingen: Stauffenburg, 5-20. 


\section{ANEXOS}

\section{Anexo 1 - Encuesta 1/2010}

Estudio empírico-experimental del proceso translativo en traducción inversa Grupo de investigación TRADYTERM

\begin{tabular}{|c|c|c|}
\hline \multicolumn{3}{|c|}{ DATOS DEL SUJETO } \\
\hline $\begin{array}{l}\text { Número de } \\
\text { alumno }\end{array}$ & & \\
\hline Curso & $2^{\circ}(2009 / 2010)$ & \\
\hline Sexo & Hombre $\square$ & \\
\hline Lengua C & Francés $\square \quad$ Alemán $\square$ & \\
\hline $\begin{array}{l}\text { Estancia en el } \\
\text { extranjero: }\end{array}$ & $\begin{array}{c}\text { Erasmus en un país de habla francesa } \\
\square\end{array}$ & $\begin{array}{c}\text { Erasmus en un país de habla alemana } \\
\square\end{array}$ \\
\hline
\end{tabular}

\begin{tabular}{|c|c|c|c|c|c|}
\hline \multicolumn{6}{|l|}{ CONOCIMIENTOS LENGUA C1 } \\
\hline $\begin{array}{l}\text { Indique su nivel según el MCER si } \\
\text { lo conoce }\end{array}$ & B1 & B2 & $\mathrm{C} 1$ & & $\mathrm{C} 2$ \\
\hline $\begin{array}{l}\text { Número de años de aprendizaje } \\
\text { (anterior a la formación } \\
\text { universitaria) }\end{array}$ & $<1$ año & 1 año & 2 años & 3 años & $\begin{array}{l}4 \text { años y } \\
\text { más }\end{array}$ \\
\hline Centro de aprendizaje & $\begin{array}{l}\text { Colegio/ } \\
\text { instituto } \\
\text { español }\end{array}$ & $\begin{array}{l}\text { Colegio } \\
\text { alemán/ } \\
\text { Liceo francés }\end{array}$ & $\begin{array}{l}\text { Profesor } \\
\text { particular }\end{array}$ & EOI & Academia \\
\hline
\end{tabular}

\begin{tabular}{|l|llll|}
\hline $\begin{array}{l}\text { Formación en traducción directa/ inversa ¿Ha asistido a lo largo de la formación en la UPCO } \\
\text { y/o durante la estancias ERASMUS en el extranjero a....? }\end{array}$ & Sí & $\square$ & No & $\square$ \\
\hline Clases de teoría de la traducción & Sí & $\square$ & No & $\square$ \\
\hline Clases de análisis de texto aplicado a la traducción & Sí & $\square$ & No & $\square$ \\
\hline Clases sobre aspectos procesuales de la traducción & Sí & $\square$ & No & $\square$ \\
\hline $\begin{array}{l}\text { Clases prácticas de traducción (inversa y o directa) en } \\
\text { las que se abordaron cuestiones relativas a estrategias } \\
\text { de traducción }\end{array}$ & & & & \\
\hline
\end{tabular}

\begin{tabular}{|l|cccc|}
\hline Macroestrategia de traducción I & & & \\
\hline $\begin{array}{l}\text { Leo primero el TO en su totalidad y empiezo a } \\
\text { traducir }\end{array}$ & Sí & $\square$ & No & $\square$ \\
\hline $\begin{array}{l}\text { Leo el TO previamente en su totalidad, identificando } \\
\text { las partes del TO que representan un problema para } \\
\text { mí }\end{array}$ & Sí & $\square$ & No & $\square$ \\
\hline $\begin{array}{l}\text { Leo el TO previamente en su totalidad, identificando } \\
\text { las partes del TO que representan un problema para } \\
\text { mí y busco posibles soluciones antes de empezar la } \\
\text { traducción del texto en su conjunto }\end{array}$ & Sí & $\square$ & No & $\square$ \\
\hline $\begin{array}{l}\text { Echo una ojeada al TO para saber de qué trata, pero } \\
\text { no hago una lectura previa de todo el texto y empiezo } \\
\text { a traducir por la primera frase }\end{array}$ & Sí & $\square$ & No & $\square$ \\
\hline Empiezo directamente a traducir por la primera frase & Sí & $\square$ & No & $\square$ \\
\hline
\end{tabular}




\begin{tabular}{|l|ccc|}
\hline Macroestrategia de traducción II & Sí & & \\
\hline $\begin{array}{l}\text { a) Empiezo la traducción del TO siempre por el } \\
\text { principio del texto }\end{array}$ & Sí & $\square$ & No \\
\hline $\begin{array}{l}\text { b) Para empezar la traducción del TO elijo un } \\
\text { fragmento del texto, pero no necesariamente desde el } \\
\text { principio del TO }\end{array}$ & & & $\square$ \\
\hline $\begin{array}{l}\text { Si ha respondido b) } \\
\text { ¿Qué fragmento elige y porqué? }\end{array}$ & Sí & $\square$ & No \\
\hline
\end{tabular}

\begin{tabular}{|l|cccc|}
\hline Macroestrategia de traducción III & & & \\
\hline $\begin{array}{l}\text { Mi forma de abordar la traducción de un TO es la } \\
\text { misma en la traducción directa y la inversa }\end{array}$ & Sí & $\square$ \\
\hline $\begin{array}{l}\text { Si ha respondido no ¿En qué se diferencian? ¿Cómo } \\
\text { procede en la directa como en la inversa? }\end{array}$ & Sí & $\square$ & No & $\square$ \\
\hline
\end{tabular}

\begin{tabular}{|l|cccc|}
\hline Unidades de traducción ¿Cuál es la unidad de traducción que enfoca a la hora de traducir? \\
\hline $\begin{array}{l}\text { La unidad básica es una oración desde su principio } \\
\text { hasta el punto. }\end{array}$ & Sí & & No \\
\hline $\begin{array}{l}\text { Traduzco de un tirón unidades más cortas que una } \\
\text { oración completa. }\end{array}$ & Sí & $\square$ & No \\
\hline La unidad básica es el párrafo. & Sí & $\square$ & No & $\square$ \\
\hline No tengo conciencia de cómo suelo proceder. & Sí & $\square$ & No & $\square$ \\
\hline $\begin{array}{l}\text { Mi forma de proceder en la tarea traslativa es la } \\
\text { misma en la traducción directa e inversa }\end{array}$ & Sí & $\square$ & No & $\square$ \\
\hline
\end{tabular}

\begin{tabular}{|c|c|c|c|c|}
\hline \multicolumn{5}{|l|}{ Macroestrategia de revisión } \\
\hline $\begin{array}{l}\text { Traduzco el TO en su totalidad y al final reviso la } \\
\text { traducción. }\end{array}$ & Sí & $\square$ & No & $\square$ \\
\hline $\begin{array}{l}\text { Traduzco por párrafos y reviso cada párrafo } \\
\text { antes de seguir con la traducción. }\end{array}$ & Sí & $\square$ & No & $\square$ \\
\hline $\begin{array}{l}\text { Traduzco una oración o fragmentos más cortos y } \\
\text { reviso a continuación. }\end{array}$ & Sí & $\square$ & No & $\square$ \\
\hline No reviso la traducción al finalizar la tarea. & Sí & $\square$ & No & $\square$ \\
\hline Al revisar la traducción, la comparo con el TO. & Sí & $\square$ & No & $\square$ \\
\hline $\begin{array}{l}\text { Al revisar la traducción, solo me fijo en el texto meta, } \\
\text { no la suelo comparar con el TO }\end{array}$ & Sí & $\square$ & No & $\square$ \\
\hline \multicolumn{5}{|l|}{ Búsquedas en la www } \\
\hline ¿Ha buscado en la www? & Sí & $\square$ & No & $\square$ \\
\hline \multicolumn{5}{|l|}{ ¿Qué tipo de información ha buscado? } \\
\hline $\begin{array}{l}\text { He buscado información para resolver problemas } \\
\text { léxicos (equivalentes; sinónimos). }\end{array}$ & Sí & $\square$ & No & $\square$ \\
\hline He buscado información de carácter enciclopédico. & Sí & $\square$ & No & $\square$ \\
\hline $\begin{array}{l}\text { He buscado información acerca del significado de una } \\
\text { palabra en TO. }\end{array}$ & Sí & $\square$ & No & $\square$ \\
\hline He buscado información de índole gramatical. & Sí & $\square$ & No & $\square$ \\
\hline
\end{tabular}

\begin{tabular}{|l|cccc|}
\hline Percepción de la competencia traductora y de la dificultad del TO & & \\
\hline $\begin{array}{l}\text { ¿Cómo calificaría su competencia traductora en } \\
\text { lengua C? }\end{array}$ & Sí & $\square$ & No & $\square$ \\
\hline ¿Cómo calificaría el nivel de dificultad del TO? & Sí & $\square$ & No & $\square$ \\
\hline
\end{tabular}




\begin{tabular}{|l|l|}
\hline \multicolumn{2}{|l|}{ Problemas de traducción del TO } \\
\hline $\begin{array}{l}\text { Señale en el TO los segmentos que han supuesto un } \\
\text { problema de traducción para Ud. y diga porqué }\end{array}$ & Motivo de la dificultad \\
\hline $\begin{array}{l}\text { La auténtica historia de la @ } \\
\text { (Ver nota ¿??- Desde "La mayor parte" hasta "del } \\
\text { correo electrónico" - ¿? palabras) }\end{array}$ & \\
\hline
\end{tabular}

\section{Anexo 2 - Encuesta 2/2011}

Estudio empírico-experimental del proceso translativo en traducción inversa Grupo de investigación TRADYTERM

\begin{tabular}{|l|ll|}
\hline DATOS DEL SUJETO \\
\hline Número de alumno & \\
\hline Curso & $3^{\circ}(2010 / 2011)$ & \\
\hline Sexo & Mujer $\quad \square$ & Hombre $\quad \square$ \\
\hline
\end{tabular}

\begin{tabular}{|l|llll|}
\hline FORMACIÓN & Sí & $\square$ & No & $\square$ \\
\hline $\begin{array}{l}\text { ¿Ha cursado la asignatura } \\
\text { Teoría e Historia de la Traducción? }\end{array}$ & Francés & $\square$ & Alemán $\quad \square$ \\
\hline Lengua C & & & \\
\hline
\end{tabular}

\begin{tabular}{|l|cccc|}
\hline Macroestrategia de traducción I & & & & \\
\hline Leo primero el TO en su totalidad y empiezo a traducir & Sí & $\square$ & No & $\square$ \\
\hline $\begin{array}{l}\text { Leo el TO previamente en su totalidad, identificando las } \\
\text { partes del TO que representan un problema para mí }\end{array}$ & Sí & $\square$ & No & $\square$ \\
\hline $\begin{array}{l}\text { Leo el TO previamente en su totalidad, identificando las } \\
\text { partes del TO que representan un problema para mí y } \\
\text { busco posibles soluciones antes de empezar la traducción } \\
\text { del texto en su conjunto }\end{array}$ & Sí & $\square$ & No & $\square$ \\
\hline $\begin{array}{l}\text { Echo una ojeada al TO para saber de qué trata, pero no } \\
\text { hago una lectura previa de todo el texto y empiezo a } \\
\text { traducir por la primera frase }\end{array}$ & Sí & $\square$ & No & $\square$ \\
\hline Empiezo directamente a traducir por la primera frase & Sí & $\square$ & No & $\square$ \\
\hline
\end{tabular}

\begin{tabular}{|l|cccc|}
\hline Macroestrategia de traducción II & & & & \\
\hline $\begin{array}{l}\text { a) Empiezo la traducción del TO siempre por el principio } \\
\text { del texto }\end{array}$ & Sí & $\square$ & No & $\square$ \\
\hline $\begin{array}{l}\text { b) Para empezar la traducción del TO elijo un fragmento } \\
\text { del texto, pero no necesariamente desde el principio del } \\
\text { TO }\end{array}$ & Sí & $\square$ & No & $\square$ \\
\hline $\begin{array}{l}\text { Si ha respondido b) } \\
\text { ¿Qué fragmento elige y porqué? }\end{array}$ & & & & \\
\hline
\end{tabular}

\begin{tabular}{|l|lll|}
\hline Macroestrategia de traducción III & & & \\
\hline $\begin{array}{l}\text { Mi forma de abordar la traducción de un TO es la misma } \\
\text { en la traducción directa y la inversa. }\end{array}$ & Sí & $\square$ & No \\
\hline $\begin{array}{l}\text { Si ha respondido no ¿En qué se diferencian? ¿Cómo } \\
\text { procede en la directa como en la inversa? }\end{array}$ & & \\
\hline
\end{tabular}




\begin{tabular}{|c|c|c|c|c|}
\hline \multicolumn{5}{|l|}{ Macroestrategia de revisión } \\
\hline $\begin{array}{l}\text { Traduzco el TO en su totalidad y al final reviso la } \\
\text { traducción. }\end{array}$ & Sí & $\square$ & No & $\square$ \\
\hline $\begin{array}{l}\text { Traduzco por párrafos y reviso cada párrafo } \\
\text { antes de seguir con la traducción. }\end{array}$ & Sí & $\square$ & No & $\square$ \\
\hline $\begin{array}{l}\text { Traduzco una oración o fragmentos más cortos y reviso } \\
\text { a continuación. }\end{array}$ & Sí & $\square$ & No & $\square$ \\
\hline No reviso la traducción al finalizar la tarea. & Sí & $\square$ & No & $\square$ \\
\hline Al revisar la traducción, la comparo con el TO. & Sí & $\square$ & No & $\square$ \\
\hline $\begin{array}{l}\text { Al revisar la traducción, solo me fijo en el texto meta, no } \\
\text { la suelo comparar con el TO. }\end{array}$ & Sí & $\square$ & No & $\square$ \\
\hline \multicolumn{5}{|l|}{ Búsquedas en la www } \\
\hline ¿Ha buscado en la www? & Sí & $\square$ & No & $\square$ \\
\hline \multicolumn{5}{|l|}{ ¿Qué tipo de información ha buscado? } \\
\hline $\begin{array}{l}\text { He buscado información para resolver problemas léxicos } \\
\text { (equivalentes; sinónimos). }\end{array}$ & Sí & $\square$ & No & $\square$ \\
\hline He buscado información de carácter enciclopédico. & Sí & $\square$ & No & $\square$ \\
\hline $\begin{array}{l}\text { He buscado información a cerca del significado de una } \\
\text { palabra en TO. }\end{array}$ & Sí & $\square$ & No & $\square$ \\
\hline He buscado información de índole gramatical. & Sí & $\square$ & No & $\square$ \\
\hline
\end{tabular}

\begin{tabular}{|c|c|c|c|c|c|}
\hline Competencia traductora en lengua $\mathrm{C} 1$ & \multicolumn{3}{|c|}{ INSUFICIENTE } & \multicolumn{2}{|c|}{ EXCELENTE } \\
\hline $\begin{array}{l}\text { ¿Cómo calificaría su competencia } \\
\text { traductora en lengua meta C? }\end{array}$ & 1 & 2 & 3 & 4 & 5 \\
\hline $\begin{array}{l}\text { ¿Cree que posee una mayor competenci } \\
\text { traductora en C que el curso anterior? }\end{array}$ & & & & & \\
\hline $\begin{array}{l}\text { ¿Cómo calificaría el nivel de dificultad } \\
\text { del TO? }\end{array}$ & 1 & 2 & 3 & 4 & 5 \\
\hline & $\begin{array}{l}\text { Muy } \\
\text { Fácil }\end{array}$ & fácil & $\begin{array}{l}\text { Dificul. } \\
\text { Media }\end{array}$ & Difícil & $\begin{array}{l}\text { Muy } \\
\text { Díficil }\end{array}$ \\
\hline
\end{tabular}

\begin{tabular}{|l|l|}
\hline Problemas de traducción del TO \\
\hline $\begin{array}{l}\text { Señale en el TO los segmentos que han supuesto un problema de } \\
\text { traducción para Ud. y diga porqué }\end{array}$ & Motivo de la dificultad \\
\hline $\begin{array}{l}\text { HISTORIA DE LA PATATA } \\
\text { (Ver nota } \\
-\end{array}$ ? - Desde "La historia de la patata" hasta "la tortilla" & \\
$-i$ ? palabras) & \\
\hline
\end{tabular}

\section{Anexo 3 - Texto origen experimento 2010}

Fuente del texto original: ABC del 20 de abril de 2010

http://www.abc.es/20100420/ciencia-tecnologia-tecnologia-internet/autentica-historia-201004201807. html

\section{Texto origen modificado y adaptado}

\section{La auténtica historia dela @}

La mayor parte de los internautas desconocen el origen y el significado de este raro pero ubicuo carácter. Dado su utilización actual, casi siempre ligada al ámbito de los ordenadores o del correo electrónico, se podría pensar que se trata de un símbolo especialmente concebido para ese uso, con una antigüedad no mayor a un par de décadas. Pero lo cierto es que se trata de un símbolo antiguo, conocido y utilizado en la Edad Media, hace más de 500 años. 
La mayoría de los historiadores aceptan que el origen de la palabra «arroba» proviene del idioma árabe, concretamente del término «ar-roub», que significa cuatro. En cuanto al símbolo en sí mismo, esa especie de «a» encerrada por un círculo, tiene sus orígenes en una práctica común entre los encargados de copiar libros en latín, a mano, allá por la Edad Media. Estos copistas utilizaban @, uniendo entre sí las letras «a»y «d» para formar la preposición latina «ad», que significa «hasta»o «hacia». Poco a poco, la @ fue haciéndose popular en otros ámbitos, y empezó a aparecer en las cartas oficiales redactadas en latín antes del nombre de su destinatario.

Pero con el paso del tiempo la @ dejó de utilizarse. Solamente se mantuvo en los Estados unidos, donde se empleaba en los registros contables, estableciendo el precio unitario de un producto en una factura. Esto hizo que cuando se inventó la máquina de escribir, a fines del siglo XIX, el símbolo de la arroba fuese incluido en su teclado. Y, como el teclado de los ordenadores es una evolución de los de aquellas máquinas, la arroba también se encuentra en ellos.

La relación de la @ con el correo electrónico, en cambio, es muy posterior a todo esto. Cuando el ingeniero Ray Tomlinson, que creó el e-mail en 1971, estaba buscando un símbolo que sirviese para identificar las direcciones de correo, uno de los pocos disponibles en los teclados era justamente la arroba. De esa manera, un carácter inventado por los copistas medievales como una forma de simplificar su trabajo se convirtió en el símbolo del correo electrónico. (358 palabras)

\section{Anexo 4 - Texto origen experimento 2011}

Fuente del texto original: http://www.todopatatas.com/historia.php Autor: Jorge Fernández Nogueira

\section{Texto origen modificado y adaptado}

\section{Historia de la patata}

La historia de la patata está rodeada de muchos misterios y su rápida expansión desde el nuevo mundo a la vieja Europa tiene espacios y lagunas que conforman capítulos a medio camino entre la crónica real y la leyenda transmitida.

Las primeras patatas llegaron a España en 1560 a manos de Pedro Cieza de León. Fueron presentadas a Carlos I y algunos ejemplares se enviaron al papa Julio II como curiosidad botánica que florecieron en los jardines de Roma durante muchos años.

La patata se usó principalmente como planta ornamental de jardines y patios, hasta que en la Europa de los siglos XVI y XVII surgió el debate sobre el consumo de las patatas como alimento. Unos le atribuyeron propiedades medicinales y afrodisíacas. Otros la acusaban de ser la causante de muchas enfermedades, entre ellas la lepra, al pertenecer a la familia de las solanáceas que son plantas con componentes venenosos. Esto hizo que durante muchos años permaneciera apartada de las mesas europeas por ser considerada maléfica y de rápida reproducción. Pero fue un farmacéutico francés, Antonio Augusto Parmentier, quien la dio a conocer como gesto de agradecimiento al sobrevivir gracias a ella cuando fue hecho prisionero por los prusianos.

La patata fue un elemento muy importante en la industrialización europea. Esto tiene su fundamento histórico en que la patata vino a ser la salvación de la alimentación de la plebe europea cuando estaba en vísperas de convertirse en el proletariado preindustrial, pues en Europa no había entonces un alimento capaz de sostener por sí sólo una jornada de trabajo. Rusia palió sus hambrunas con la patata, de modo que su diezmada población hizo suyo el cultivo de la patata y de ella obtuvieron su bebida nacional: el Vodka.

Al margen de su historia y de sus propiedades alimenticias, la otra gran cualidad de la patata es la diversidad de formas de preparación que admite tanto como plato único como de acompañamiento de otros ingredientes principales. Pero hay dos formas de prepararla que destacan de las otras, una presumiblemente anglosajona, las patatas fritas o "chips" y otra española, "la tortilla". (354 palabras) 